\title{
Tuberculosis care in foreigners: ethical considerations
}

\author{
E. Tala
}

In the past, a disease was strictly confidential between the patient and the doctor. However, even primitive society laid special rules for certain dangerous diseases, and patients had to follow these regulations but without receiving any support on the part of society; on the contrary, these people were unhappy, socially isolated, and without the rights of healthy people.

Over the centuries, a long process has taken place and different types of societies with different rules have been developed. In this process, individual entitlements have increasingly been interfered with by the third party, society, and its rights. At present, whereas the poorest communities cannot even afford a system of basic health care, in technically advanced countries the full range of sophisticated modern medicine is available for community members. Ethically, however, all members of any society - first world, third world - should have equal rights to health and equal access to health services. The discrimination of diseased persons is unethical. Therefore, the concepts of health and of disease are intimately linked with structure of culture and society.

However, people coming from outside, with a completely different set of values and having a different cultural background, simply cannot immediately and without difficulties adapt themselves to a new environment in a community strange to them. This is the key question when we plan health care provision for foreign people.

Only a few countries impose health conditions, in general, before granting an entrance visa to the country, either as a formality, or requiring a proper doctor's certificate or health examination. In this case, also, the situation is different if only a limited number of applications have to be handled, or if there are large numbers of foreign people queuing in a border station.

There are no fixed supranational rules for entrance, either ethical or legislative. The United Nations Charter from 1945 contains some general points concerning international humanitarian co-operation. In the European Union, the Maastricht Treaty, in force since November 1, 1994, will govern Community policy for the coming years, including the health sector. The member states of the European Union (EU) will follow a common refugee policy, which is intended to restrict the entrance of refugees to EU countries. There is also a European Community Working Group on Health Services [1]. At present, however, in practice, national legislation, rules

Dept of Diseases of the Chest, University of Turku, FIN-21540 Preitilä, Finland. and regulations still have to be followed. On the other hand, an important role is going to be played by recommendations, agreements, or consensus reports; and the higher the authority behind them, the more impact they will have. For example, the position paper, approved at the second Wolfheze Meeting, of the International Union Against Tuberculosis and Lung Disease (IUATLD) and the World Health Organization (WHO) in March 1994, which is published in this issue [2], is intended to be an important document in steering ethical and practical attitudes on tuberculosis control among foreign people.

International migration has long been, and continues to be, a major phenomenon of our times. Foreign people have many problems needing to be solved outside the health sector, e.g. bad housing, overcrowding, low income, unemployment, discrimination, and the sense of belonging to an underclass. Other problems arise from cultural and educational differences; and, consequently, there are many barriers to be overcome [3].

The first and very important challenge is the language barrier; but equally problematical is a different way of thinking, the difficulty of differentiating between facts and feelings, inability to understand our bureaucratic health care system and the jargon we are familiar with. One of the reasons for misunderstandings can be that both partners have different meanings for "illness" and "contagious diseases" in their own frames of reference. For example, foreign people may have a pluralistic medical system, where the same disease may have several different causes, often linked with religious frames.

Religious values may be even more important than health, and can be the reason for refusal of medical services. Also, preference may be given to traditional folk healers and strong medicines, rather than to the laboratory and X-ray examinations which we consider necessary before, for example, tuberculosis chemotherapy. Some refugees may have been subjected to torture, as a result of which they may be afraid of even simple measures, such as approaching an X-ray machine, needles and so on. Therefore, the understanding of language, and a knowledge of the patient's personal history and cultural background, are the keys that may best resolve misunderstandings.

As mentioned earlier, contagious diseases are a special issue, where society has long ago taken steps to prevent the spread of disease and to protect, by rules or legislation, the healthy members of society from illness [4]. Today, society has also often taken responsibility for arranging treatment, free of charge, and in many countries even against the patient's will, although this is 
rarely the case. Tuberculosis is a good example of this type of disease, linked mutually with the patient's and with society's rights. However, in practice, the legislation and regulations laid down in the recipient country for their own inhabitants have to be followed for foreign people as well. It is important that indigenous and foreign people should have equal rights and duties. It is necessary to ensure that foreign people understand that effective treatment is possible, which may not have been the case in their country of origin, and it must be ensured that health examination is never used as the grounds for denying admission to a country.

As clearly demonstrated in the European Task Force document [2], tuberculosis is one of the most important diseases among foreign populations; moreover, in many European countries, foreign-born persons represent about a half, or even more, of newly notified cases of tuberculosis, even though they constitute only a small minority of the population [5]. Tuberculosis is a contagious disease, and we also know that people once infected with tubercle bacilli have a lifetime risk of contracting the illness. Consequently, we have an ethical and moral duty to prevent the spread of infection, all the more so because we have the ethically acceptable means for doing it. We have to treat tuberculosis, because it is a curable disease; moreover, it can be treated very cost-effectively, and so we may relieve human suffering and avoid premature deaths $[6,7]$. Therefore, it is ethically fully justified to include foreign people immediately within the tuberculosis control programme. A wide consensus on programme elements is needed, and the Position paper adopted at Wolfheze by a European Task Force offers a valuable basis for international application [2]
Hopefully, the resulting recommendations will be backed up by a strong political will. However, if we hide behind ethical considerations, forget the hard scientific data on tuberculosis, and do little or nothing about the effective application of present knowledge to the benefit of the human race, we risk the curse of future generations. The important challenge and great opportunity to treat tuberculosis independently of the person's ethnic origin, is upon us now.

\section{References}

1. Anonymous. Health issues for Europe (Editorial). Lancet 1994; 343: 245-246.

2. Rieder HL, Zellweger J-P, Raviglione MC, Keizer ST, Migliori GB. Report of a European Task Force. Tuberculosis control in Europe and international migration. Eur Respir J 1994; 7: 1546-1554.

3. Tala E. Migration, ethnic minorities and tuberculosis. Eur Respir J 1989; 2: 492-493.

4. Higginson J, Chu F. Ethical considerations and responsibilities in communicating health risk information. $J$ Clin Epidemiol 1991; 44: 51S-56S.

5. Clancy L, Rieder HL, Enarson DA, Spinaci S. Tuberculosis elimination in the countries of Europe and other industrialized countries. Eur Respir J 1991; 4: 12881295.

6. Rider HL. Misbehaviour of a dying epidemic: a call for less speculation and better surveillance. Tubercle Lung Dis 1992; 73: 181-183.

7. Murray CJL. Resource allocation priorities: value for money in tuberculosis control. In: Porter JDH, McAdam KPWJ, eds. Tuberculosis; Back to the Future. Chichester, England, John Wiley \& Sons, 1994; pp. 193-208. 